\title{
Patella Alta and Trochlea Dysplasia Is Associated with Abnormal Type II Collagen and Matrix Accumulation in Chondrocytes
}

\author{
Peter Storgaard Skagen ${ }^{*}$, Thomas Horn², Aubrey Milunsky³, David Dejour ${ }^{4}$, \\ Bente Stærgaard ${ }^{2}$, Hanne Aagaard Kruse ${ }^{2}$, Tom Nicolaisen ${ }^{1}$ \\ ${ }^{1}$ Department of Sports Traumatology, Frederikssund Hospital, Frederikssund, Denmark \\ ${ }^{2}$ Department of Pathology, Herlev University Hospital, Herlev, Denmark \\ ${ }^{3}$ Boston University School of Medicine, Center for Human Genetics, Boston, USA \\ ${ }^{4}$ Department of Orthopaedic \& Sportmedicine Surgery Lyon-Ortho-Clinic, Lyon, France \\ Email: *peterskagen@yahoo.dk
}

Received 14 January 2014; revised 16 February 2014; accepted 31 March 2014

Copyright @ 2014 by authors and Scientific Research Publishing Inc.

This work is licensed under the Creative Commons Attribution International License (CC BY). http://creativecommons.org/licenses/by/4.0/

c. (i) Open Access

\section{Abstract}

The purpose of this study was to perform morphological and molecular analyses of articular cartilage from a 14-year-old boy with unusual cartilage lesions, patella alta and trochlea dysplasia in both knee joints and clinically examine two family members (sister, mother), also affected in their knee joints. Biopsies from the boy's patella were used for: histological examination, Transmission Electron Microscopy (TEM) and DNA sequencing of the COL2A1 gene including Multiplex Ligation-dependent Probe Amplification (MLPA), for detection of DNA deletions and duplications. Clinical and radiological examination showed patella alta and trochlea dysplasia for the brother (type D), sister (type A) and mother (type A) with Insall-Salvati ratios of 1.50, 1.46 and 1.3. Light Microscopy (LM) of biopsies from the patient showed rhomboid chondrocytes in lacuna with deposition of protein aggregates in the ECM. TEM revealed abnormal type II collagen fibrils in aggregates and chondrocytes with abnormal matrix accumulation in rough Endoplasmic Reticulum (rER). Immunostaining showed that type II collagen was deposited intracellularly and in protein aggregates, together with type I collagen, indicating alterations in chondrocyte function and turnover of these molecules. DNA sequencing of 54 exons including extended DNA analysis with MLPA was non-conclusive. Conclusions: We suggest that patella alta and trochlea dysplasia for this patient is associated with collagen accumulation in chondrocytes, abnormal type II collagen heterofibrils in the ECM, cell death and cartilage with subnormal strength and increased risk of premature patellofemoral arthritis. A family with these disorders suggests that phenotype might be

\footnotetext{
"Corresponding author.
} 


\title{
transmitted as an autosomal dominant trait.
}

\section{Keywords}

\author{
Patella Alta; Trochlea Dysplasia; Matrix Accumulation; Endoplasmic Reticulum Storage Disease \\ (ERSD)
}

\section{Introduction}

Chondrodysplasia is a subset of skeletal dysplasia affecting chondrogenesis development and can result in an abnormal skeleton and dwarfism, visual or hearing impairment, facial abnormalities, dwarfism and joint problems, including idiopathic cartilage lesions which can develop into early-onset osteoarthritis [1]. Recently, identification of disease related genes has contributed significantly in our understanding of these abnormalities. Today, more than 100 different COL2A1 human mutations have been described and listed in the Cardiff University Human Gene mutation database (http://www.hgmd.org) and these mutations give rise to a clinical spectrum of autosomal dominant conditions-the type II collagenopathies.

Clinical phenotypes associated with type II collagenopathies, which account for the majority of cartilage diseases in humans, range from 1) severe prenatally lethal and short stature conditions such as achondrogenesis and hypochondrogenesis, 2) severe-moderate conditions such as; Stickler syndrome, Kniest dysplasia, SpondyloEpiphyseal-Dysplasia (SED), Multiple Epiphyseal Dysplasia (MED), and generalised arthritis to 3) mild clinical phenotypes such as premature osteoarthritis [2], which may be apparent only in adulthood.

In order to improve our biological and pathological understanding of "mild" clinical phenotypes, we have initiated clinical investigations of idiopathic cartilage lesions in young people's knee joints. In particular, we have focused on cartilage lesions involving juvenile Osteo Chondritis Dissecans (OCD) [3] and patellofemoral disorders. Especially, developmental and familial abnormalities such as patellofemoral dysplasia which include patella alta, trochlea dysplasia and chondromalacia patella in young people and children, are often unrecognized cases in daily practice and may, therefore, be diagnosed as common orthopedic diseases or not be diagnosed at all.

Surprisingly, analyses of cartilage biopsies from patients with OCD and patella alta/trochlea dysplasia have demonstrated similar chondrocyte phenotypes such as strong abnormal protein accumulation in rough endoplasmic reticulum (rER) and cell death, a characteristic feature which is similar to genetically inherited Endoplasmic Reticulum Storage Diseases (ERSD) such as osteogenesis imperfecta (OI), congenital hypothyroid goiter, familial hypercholesterolemia and the collagenopathies [4].

Patella alta refers to an abnormally high standing patella in relation to the femoral condyles resulting in the patella riding too high in the trochlear groove. Knees with patella alta have reduced patellar contact areas when compared with knees of normal patellar height, and these reduced patellar contact areas lead to greater patellofemoral stress on these areas, for instance during fast walking [5].

In a large investigation of patients with recurrent dislocation of the patella, Thestrup Andersen [5] found that 207 out of 286 knee joints had patella alta. In addition, patella alta has been reported to have a significant presence in knees with osteoarthritis (OA) when compared to those with a normal femoral-patellar joint [6].

It is not known whether patella alta is an inherited or acquired trait, but patella alta has been implicated as a cause of chondromalacia [7] as well as being associated with Osgood-Schlatter disease and Sinding-Larsen Johansson's disease [8] [9]. Further, patella alta is rarely an isolated finding and is most often part of a regional patello femoral dysplasia [10]. David Dejour and Le Coultre [11] found that approximately 96\% of patients with a history of true patellar dislocation had evidence of trochlear dysplasia, suggesting that patellar instability could be a congenital disorder.

Even though patella alta has been known since 1899, where Schulthess [12] described some spastics with high riding patella, to our knowledge, this is the first report which describes an association between patella alta and trochlea dysplasia in relation to abnormal type II collagen hetero-fibrils and matrix accumulation in chondrocytes. 


\section{Methods}

\subsection{Patient History}

The patient was a 14 years old boy. He was referred to the clinic as a sub-acute case because he had very severe pain and marked swelling in his right knee. There had been no trauma. For a couple of years he had periods with light to moderate retropatellar pain in both knees which was more pronounced in the right knee after strenuous activities. For the last 3 - 4 months before he was seen in the clinic, his symptoms became more severe in the right knee. His pain was intense and often the knee was swollen and he experienced locking of the right knee several times a week-lasting up to several hours.

Apart from the knee, the patient was in good health and had developed normally though very fast in his early puberty. At the time of referral he was $175 \mathrm{~cm}$ high and weighted $60 \mathrm{~kg}$. He had been conceived by In Vitro Fertilization (IVF).

When he was examined at the clinic, the knee was swollen, extremely tender and with a large volume of effusion. There was severe retro-patellar pain. X-ray pictures showed that his tibias were normally developed, while his patellas and trochlea on both knees were dysplastic and patella alta was present (Figure 1(A)).

Arthroscopy showed that the patella cartilage was severely damaged with fragments of cartilage were detaching from the cartilage surface. In the arthroscopic view, the patella and trochlea were dysplastic and laterally subluxated and tightly bound laterally. The flakes of cartilage were removed with forceps and shaver and a lateral release was performed, to allow the patella to centre more anatomically in the trochlea. The largest flakes of cartilage from the patella were used for further investigations.

During the following years, he continued with suitable physiotherapy and his symptoms gradually diminished and eventually disappeared in activities of daily life.

\subsection{Family History}

The father (age 51) reported never having experienced knee problems, but the mother (age 49) had to give up all sports activity at the age of twenty years because of pain in both of her knee joints. The patient's sister (age 15 at this time) was also troubled by pain in both knee joints.

The mother and sister were clinically examined in the clinic (Figure 2) and lateral radiographs (X-ray) (Figure 1) were taken in the early summer of 2010 of both knee joints. Their symptoms, however, were not severe

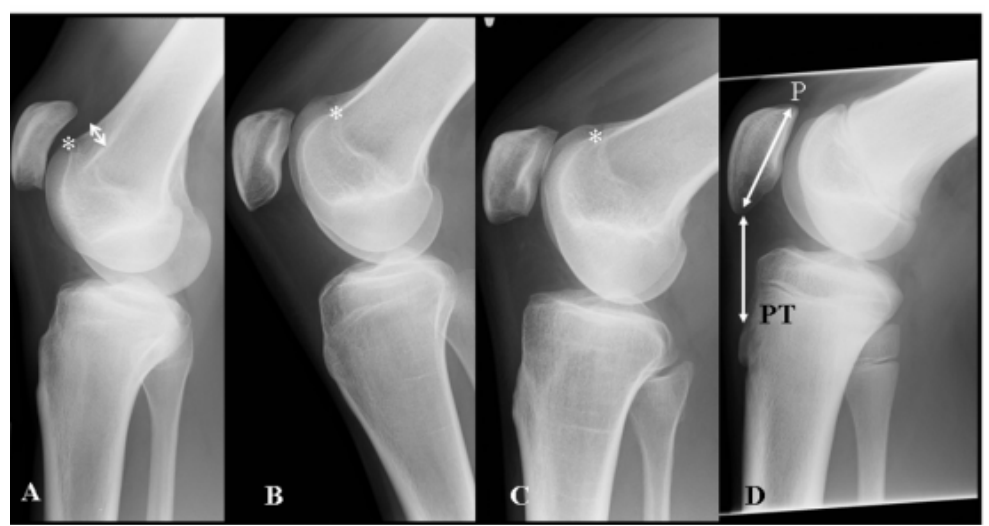

Figure 1. X-ray pictures from early summer 2010, 6 years after our first examination of the family. The patient (A) is on the left with an Insall-Salvati ratio of 1.50 and trochlea dysplasia type $\mathrm{D}$. Note hypoplastic patella and length of patella tendon), B; sister with a ratio of 1.46 and trochlea dysplasia type A, C; mother with a ratio of 1.32 and trochlea dysplasia type A and fare right (D) is from a patient (male, age 21) with a normal configuration of the knee (Insall-Salvati ratio:1,0). On a lateral radiograph, the length of the patellar tendon (PT) should be approximately equal to the length of the patella (P) and should not be longer than 1.3 times the length of the patella. Crossing sign indicates that the trochlea is flat while the supra trochlea spur (arrow) indicates that the trochlea is prominent. 

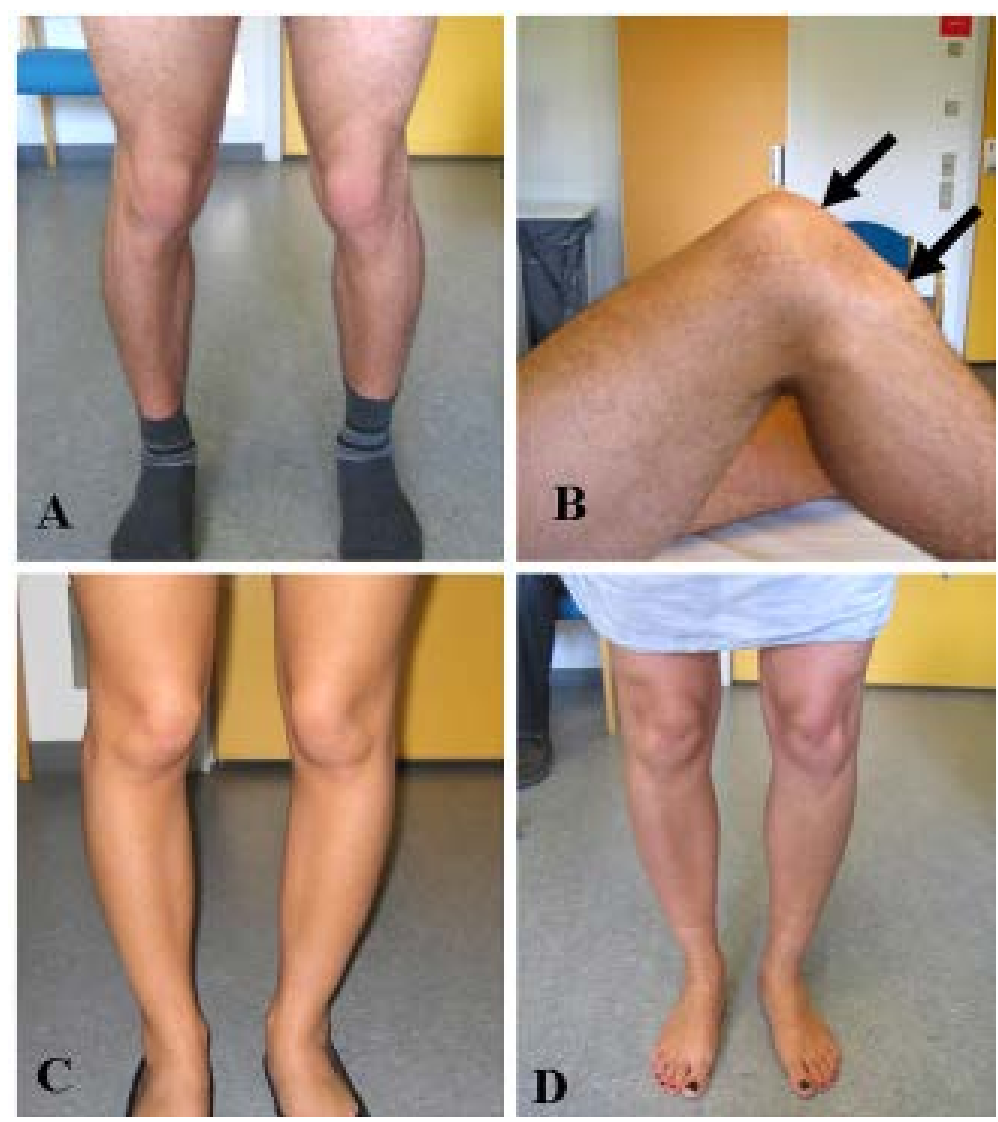

Figure 2. Pictures show the patients lower extremities at an age of $21(\mathrm{~A}+\mathrm{B})$, the sister at age 19Y (C) and their mother at age 49Y (D). Note increased Qangle, patella alta, and length of ligamentum patellae. Arrows in B points to proximal and distal end of ligamentum patella.

and did not indicate an arthroscopic examination.

\subsection{Tissue Analysis}

Cartilage pieces obtained from patella, and a blood sample obtained from the patient were supplied by Frederikssund hospital (Sports Clinic), following protocols reviewed and approved by the Danish Ethical Committee (KA-05017-GM). An informed consent was also given by the parents.

\subsection{Light Microscopy and Immunohistochemistry}

Tissue samples for LM and immunohistochemistry were prepared as described in [3].

\subsection{Explant Culturing}

Cartilage explants from patella were prepared for cell growth according to the procedure described by Skagen P et al. [3]. Explant cultured chondrocytes were used for both LM and TEM.

\subsection{Transmission Electron Microscopy}

Glutaraldehyde fixed cartilage pieces and explant cultured chondrocytes were prepared for TEM according to procedure described in [3]. The diameter of collagen fibrils present in the medium zone of cartilage pieces were carried out with Mega view III soft imaging system (Münster, Germany). Only collagen fibrils in longitudinal sections were measured. The mean diameter of fibrils and standard error (SE) of mean were determined. 


\subsection{Molecular Studies}

In the set-up for DNA analysis, we hypothesized that a family with patella alta and trochlea dysplasia was caused by mutation in type II collagen, transmitted in an autosomal dominant manner from the mother to son and daughter. We therefore sequenced the COL2A1 gene of the patient in order to understand the molecular mechanism behind the abnormal type II collagen heterofibrils and the putatively inherited dysplastic condition. Genomic DNA was isolated from blood of the patient and DNA was isolated according to a protocol developed by the manufacturer (Wizard Genomic, Promega, Madicon WI, USA). PCR amplification and automated fluorescence sequencing was performed at the Center for Human Genetics, Boston University School of Medicine, Boston, Massachusetts, USA) on all exons 1 - 54 in the COL2A1 gene. Some flanking intronic sequences (approx. 10 - $20 \mathrm{bp)}$ between adjacent exons of the gene were also sequenced.

In addition, Multiplex Ligation-dependent Probe Amplication (MLPA) analysis was set up for detection of any DNA deletions, following the directions provided by the manufacturer (MRC Holland, Amsterdam, The Netherlands). The probe set for COL2A1 covering 21 exons; 1, 1A, 4, 6, 8, 10, 16, 17, 19, 20, 24, 27, 29, 31, 35, 39, 43, 46, 49, 51 and 54.

\section{Results}

By clinical and radiological examination in the clinic, both the patient, his sister and mother showed increased Q-angle, increased length of patellar tendon and trochlear dysplasia (Figure 1 and Figure 2). Trochlea is described as dysplastic when the femoral sulcus is flattened to various degrees. We have used the classification elaborated by the Lyon school with 4 different types [11]. The patient was classified as a type D while the sister and mother both were classified as type A (Figure 1).

$\mathrm{X}$-rays and the Insall-Salvati method [13] were used to determine the position of the patella for all 3 family members. Lateral radiographs were taken with the knee flexed at 30 to 60 degrees, and the diagonal length of the patella and the length of the patellar tendon were measured. These lengths were converted into a ratio of patella length to patellar tendon length expressed as the PT/P ratio. This Insall-Salvati ratio was calculated to be 1.50 for the patient, 1.46 for the sister, and 1.32 for the mother. Normal values are between $0.8-1.2$. For comparison, a male (age 21) with a ratio of 1.0 and a normal configuration of the knee is shown in Figure 1

\subsection{Light Microscopy (Cartilage from Patient)}

Chondrocytes in biopsies were atypical with a rhomboid morphology (data not shown). Both mixtures of fibro-cartilage and hyaline cartilage were seen in the various cartilage zones. In general, several regions in the biopsies were devoid of cells (Figure 3). The focally most intense Toluidine-Blue stained region of the ECM occurred in the peri-cellular compartment of the cartilage (Figure 3(B)). Within this region, crystal-like amorphous structures were seen and numerous lacunae were empty and highly expanded (Figure 3(B)).

\subsection{Explant Cultured Chondrocytes}

In general, chondrocytes derived from cartilage explants showed high viability. Chondrocytes were rhomboid or polygonal in shape and did not resemble normal monolayer chondrocytes cultured in medium with serum as chondrocytes contained enlarged nuclei and inclusion bodies that affected morphology (Figure 4(A)).

\subsection{Immunohistochemistry}

In all zones, chondrocytes showed strong intracellular reactivity for type II collagen (Figure 4(B)) suggesting that this protein was abnormally accumulated. It was noteworthy that type I and type II collagens (data not shown) were seen in the amorphous protein aggregates and in all cartilage zones indicating that chondrocytes in the cartilage were dedifferentiated.

\subsection{TEM-Ultrastructural Characterizations}

TEM of explant cultured chondrocytes revealed that inclusion bodies were identical to electron dense large vesicles and abnormally distended rER with condensed matrix substance inside. Matrix proteins synthesized by chondrocytes showed abnormally formed collagen fibrils embedded as aggregated matrix proteins (data not shown). 

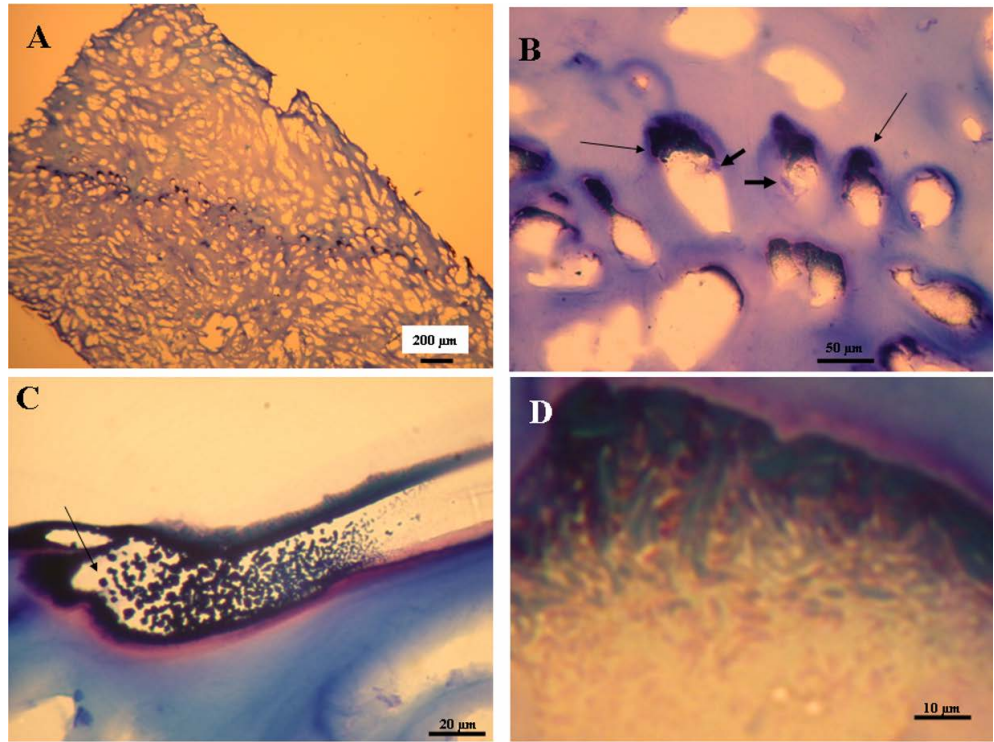

Figure 3. (A) Toluidine blue (TB) staining reveals an amorphous matrix substance of aggregates in all cartilage zones; (B) High magnification of matrix substance (thin arrows) shows that "lacuna" are empty and highly expanded. Poorly differentiated and atypical chondrocytes are also present (bold arrow); (C) Another high magnification of matrix substance with a rhomboid crystallike structure (arrow); (D) Matrix substance showing needle-like crystal morphology within the expanded "lacuna” (TB).
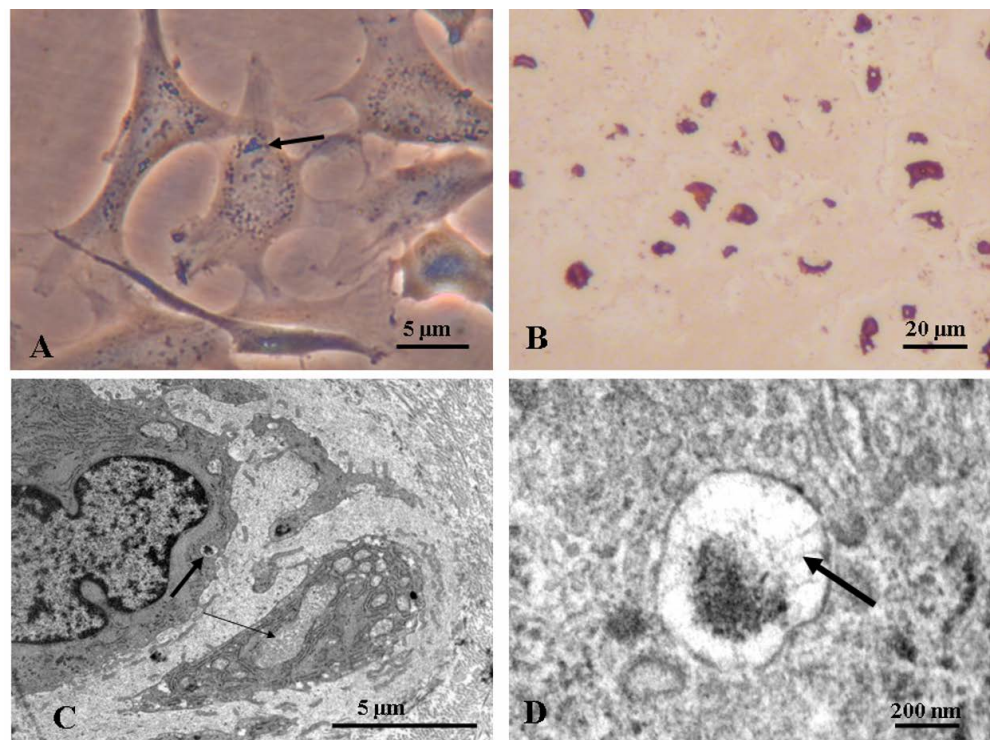

Figure 4. (A) LM shows that matrix accumulation is also present in cultured chondrocytes. Inclusions bodies (arrow) are present in cytoplasm and nuclei are enlarged (unstained); (B) Immunostaining of cartilage sections from patella with a monoclonal antibody against type II collagen. Strong staining is seen in chondrocytes indicating that type II procollagens are accumulated in the cell's organells. Large aggregates in ECM are also stained positive for type II collagens and type I collagen (data not shown); (C) TEM of chondrocytes in cartilage contains distended rER (arrow) and highly contrasted electron dense matrix substance packed in large vesicles (bold arrow); (D) High magnification TEM of vesicle in picture $C$ reveal aggregated proteins with a tread-like appeance (bold arrow). 
In comparison with chondrocytes in cartilage, these cells showed irregular hyperchromatic nuclei surrounded by intermediate filaments, and numerous secretory vesicles in the cytoplasm which represent dilated rER with accumulated matrix proteins (Figure 4(C)). In addition, cells were also vacuolated and plasma membranes (PM) showed numerous projections (filopodia) and abundant budding vesicles with matrix material released into the ECM (Figure 4(C)).

Material accumulated in rER appeared as a lamellar collection of filaments with diameters of $1-2 \mathrm{~nm}$ or as a network of aggregating matrix granules (Figure 4(D)). ECM. Typical findings in the interterritorial matrix compartment were aggregated matrix proteins and bundles composed of type II collagen hetero-fibrils running parallel (Figure 5).

In addition, the ECM was dominated by short forms (length $<300 \mathrm{~nm}$ ) and aggregates of highly atypical type II collagen hetero-fibrils (Figure 6). Short forms of collagen fibrils showed diameters of about 70 - $80 \mathrm{~nm}$, while longer $(>1000 \mathrm{~nm}$ ) type II collagen hetero-fibrils seen in aggregated areas showed diameters of $50-90 \mathrm{~nm}$ (Figure 5). Furthermore, type II collagen hetero-fibrils examined in cartilage samples did not exhibit the characteristic $(60$ - $65 \mathrm{~nm})$ D-banding pattern (Figure 6) which further implies that the staggered arrangement of collagen molecules into collagen fibrils reflected abnormal assembly.

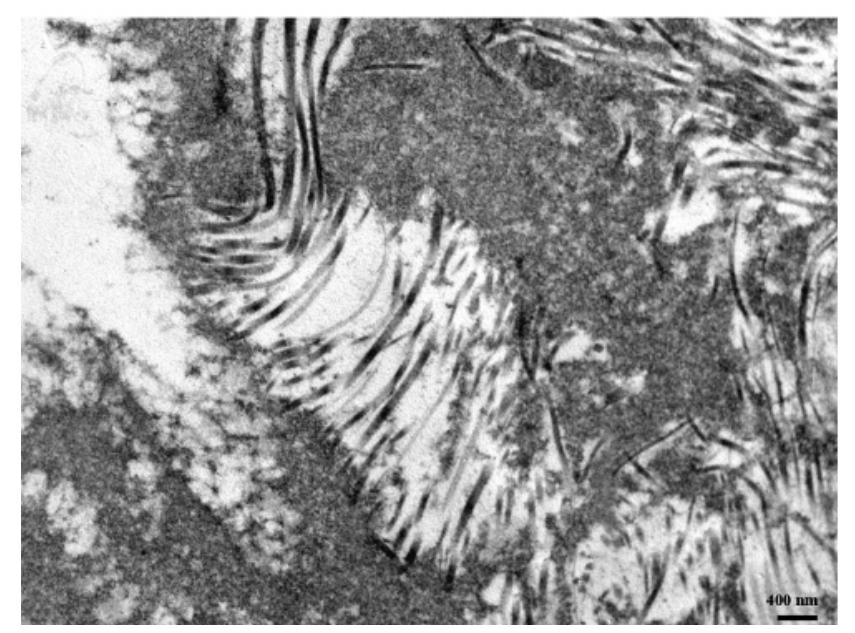

Figure 5. TEM of cartilage section shows a highly compromised ECM, composed of a network of aggregated type II collagen heterofibrils. High magnification reveals that aggregates are mainly composed of atypical type II collagen heterofibrils of various calibers and quality.

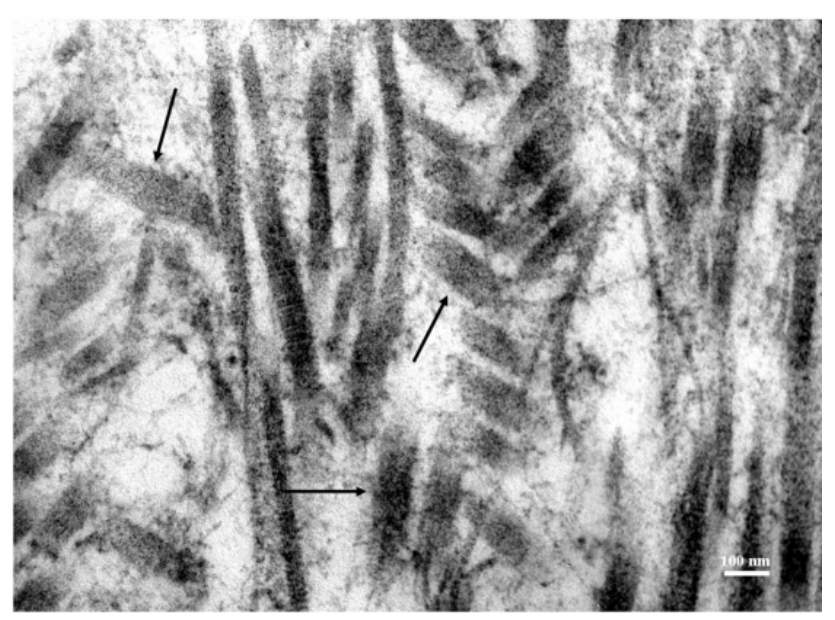

Figure 6. High magnification TEM shows "short forms" of abnormal type II collagen heterofibrils (arrows) in the ECM. 


\subsection{Molecular Analysis}

All 54 exons and some introns of the COL2A1 gene were sequenced to determine if anomalies in type II collagen heterofibrils were caused by DNA mutation. DNA sequencing of all 54 exons were found to be negative, no mutation was found within the COL2A1 gene. Only single nucleotide polymorphisms (SNP) were found: T9S $(A>T)$ in exon 1, IVS $9+15 \mathrm{~T}>\mathrm{G}$, IVS $11+15 \mathrm{G}>\mathrm{GA}$, G612G $(\mathrm{T}>\mathrm{TC})$ in exon 28, IVS $32+7 \mathrm{~A}>\mathrm{AG}$, N800N (T > TC) in exon 36, V1331I (G > GA) in exon 52, and IVS 52-14 (C > CG). As no mutation was found when we sequenced all 54 exons, we decided to expand the molecular analysis with MLDA to explore the possibility of missed intragenic deletions or duplications.

Twenty (21) exons (1, 1A, 4, 6, 8, 10, 16, 17, 19, 20, 24, 27, 29, 31, 35, 39, 43, 46, 49, 51 and 54) were analyzed via MLPA and found to be negative for any DNA deletions and duplications within the COL2A1 gene (Figure 7).

\section{Discussion}

In this paper we demonstrate that cartilage biopsies obtained from a high standing patella (patella alta) displays a compromised ECM containing; 1) short fibril forms and aggregates of abnormal type II collagen heterofibrils and 2) atypical "rhomboid" chondrocytes jeopardized by matrix accumulation in dilated rER leading to an Endoplasmic Reticulum Storage Disease (ERSD) phenotype and cell death.

With regard to the markedly abnormal cartilage phenotype, we believe that maltracking of the patella did not lead to these changes. In contrast, we suggest that the primary changes in chondrocytes and ECM leads to cartilage with subnormal strength and increased risk of premature patellofemoral arthritis. Our hypothesis may to some extent be confirmed by similar case reports, as our findings corresponds to certain human type II colla

\section{Multiplex Ligation-dependent Probe Amplification (MLBA) analysis of COL2A1 gene.}
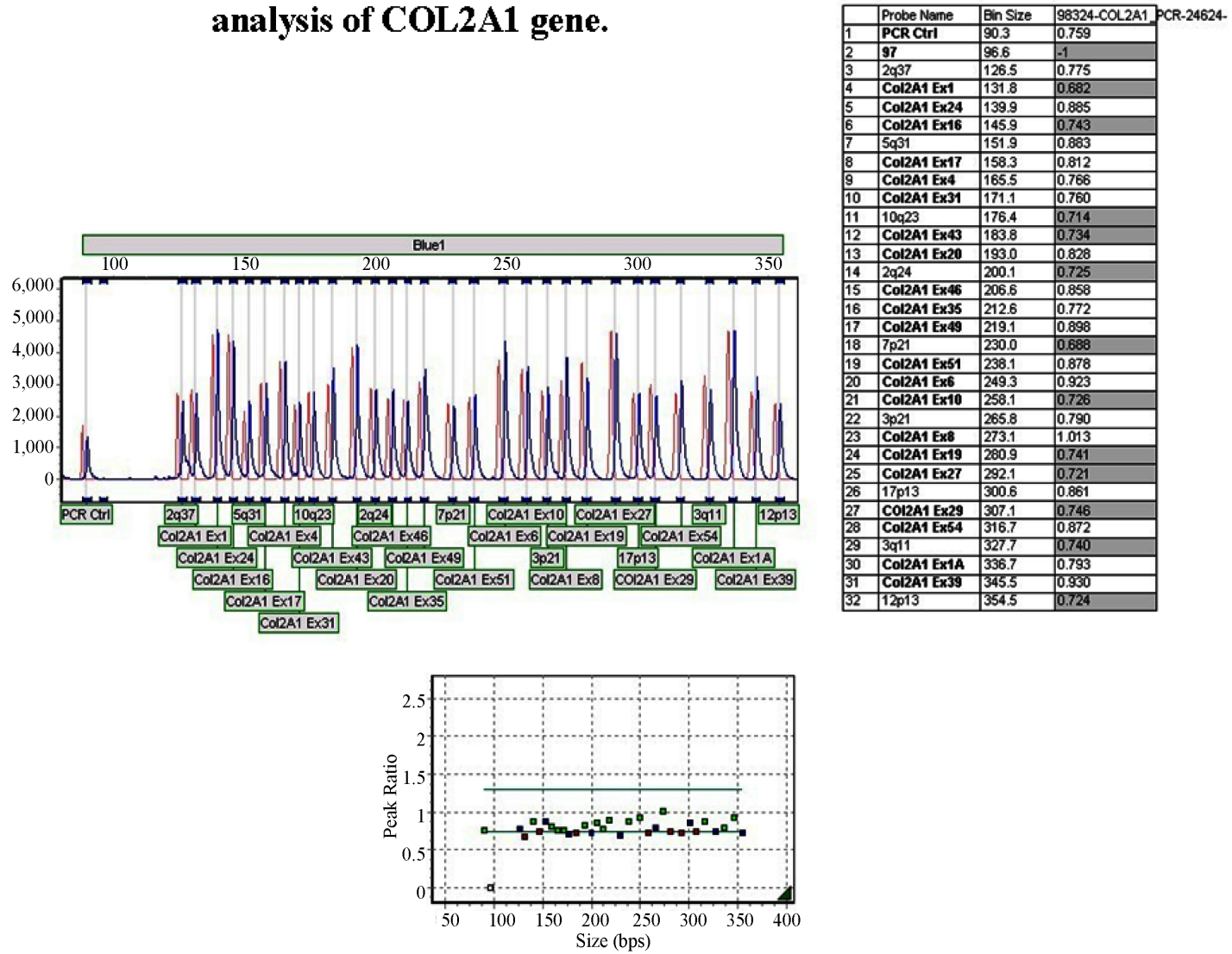

Figure 7. Multiplex ligation-dependent probe amplification analysis (MLPA) of 21 exons of the COL2A1 gene. 
genopathies [14], such as Stickler syndrome and Kniest dysplasia. These similarities are; matrix accumulation in rER in chondrocytes, abnormal type II collagen heterofibrils, formation of collagen bundles in the ECM of the cartilage and cell death [14]. These features have recently been shown also to be present in cartilage from patients with OsteoChondritis Dissecans (OCD) [3].

Immunochemistry revealed that type II collagen was present in aggregates of the ECM and was accumulated in chondrocytes suggesting that type II procollagen is misfolded in the cells which lead to intracellular protein accumulation and deposition of large protein aggregates in the peri-cellular compartments. Type I collagen was also identified with monoclonal antibodies, in the large aggregates and across the entire desiccate which is a highly atypical finding for these regions.

In comparison with other cartilage diseases, large amounts of type I collagen are also expressed at atypical regions in the articular cartilage of patients with OCD [3], achondrogenesis type II and hypochondrogenesis, the two latter diseases caused by mutations in the COL2A1 gene [15]. A phenotypical chondrocyte change such as abnormal matrix accumulation within dilated rER has also been observed. These results suggest that accumulation of (mutant) proteins does alter expression of a wide set of matrix proteins within the chondrocytes and that the chondrocytes may, in this disease process, become dedifferentiated.

With regard to the patient with patella alta, trochlea dysplasia and an abnormal cartilage of poor quality there may be a high potential to overload the contact areas which can lead to further deterioration of the cartilage and development of early OA. Indeed, patella alta has been reported to be six times as frequent in knees with osteoarthritis (OA) than in those with a normal femoral-patellar joint [6] further emphasizing that these patients might have cartilage of subnormal strength.

Patella alta is radiologically defined by an Insall-Salvati index greater than 1.2 (patella infera as < 0.8) [13]. Normal females have a significantly higher ratio than normal males (1.0878 and 1.0032 respectively) [16]. In this regard, the ratio of the length of the patellar tendon to the length of patellar was estimated to be 1.50 for the brother, 1.46 for the sister and 1.32 for the mother. These ratios are much higher values compared to normal knees or knees with clinical chondromalacia [17] or patients with Osgood-Schlatter [8]. The high index calculated for both siblings and the mother indicates that their clinical phenotypes could be similar but different from the above diseases. In addition, $x$-rays of all 3 family members (Figure 1) further indicates that they have familial chondrodysplasia with similar patella and trochlea phenotypes suggesting that hereditary might be associated with this condition.

Explant cultured chondrocytes were also shown to accumulate matrix proteins in rER and to synthesize collagen fibrils of poor quality which further supports that chondrocytes were atypical in their phenotypes. In addition, it was surprising to find that almost all chondrocytes were dead in certain areas of the biopsies. In these areas, large protein aggregates were seen (Figure 3). This phenomenon might reflect that the presence of intracellular (mutant) protein could lead to chronic activation of stress responses and activation of apoptosis thereby limiting cell survival [18]. This means that the malfolded collagen proteins could exert their effects in a dominant fashion and perturb the function of the chondrocytes that synthesize it.

The assumption that type II pro-collagen was abnormally synthesized in the chondrocytes was further sustained by TEM which showed "short forms" of abnormal type II collagen hetero-fibrils as well as longer type II collagen heterofibrils which made a network of larger aggregates in the ECM (Figure 5). In normal chondrocytes, three chains of repeating primary sequence (Gly-X-Y; amino acid residue triplets) fold into triple-helical collagen molecules with lengths of $300 \mathrm{~nm}$ and diameters of $1.5 \mathrm{~nm}$ [19]. This length corresponds almost to the length of about 200 - $250 \mathrm{~nm}$ identified for the "short" fibrils with a "normal" mean diameter of about 70-80 nm. This suggests that lateral fibril growth is controlled to an almost normal diameter while longitudinal fibril growth does not take place for numerous fibrils. This is comparable to Kniest syndrome which cause abnormally short pro- $\alpha 1$ (II) collagen chains to be produced in chondrocytes. These short chains join with longer normal-length collagen chains. The resulting abnormal type II collagen molecules are also shorter than normal. Short pro- $\alpha 1$ (II) chains are also formed in SED congenital, SED, and Stickler syndrome [14].

When making comparisons with these syndromes, there are similarities but also major differences. For instance, this patient has an isolated patella and trochlea pathology while people with Stickler, Kniest and SED typically have multiple abnormalities such as; eye problems, facial abnormalities, hearing loss, dwarfism, and joint problems [1]. Also findings such as the "network" of aggregated type II collagen heterofibrils, aggregates containing rhomboid needle-like crystals in the ECM (Figure 3(D)), high chondrocyte death (Figure 3(A)) and chondrocytes with a unique rhomboid morphology seems to be unique for this phenotype and has, to our know- 
ledge, not been described earlier. We therefore suggest that this phenotype could be a novel subfamily within the collagenopathies and that patella alta is also a condition with an elongation of the patellar tendon beyond normal, which is also a factor leading to maltracking of patella and local changes in the patellar cartilage because of increased patellofemoral contact force.

\section{Summary}

Although the extended DNA analysis did not confirm that abnormal type II collagen hetero-fibrils in the patients cartilage were related to a disease causing mutation in the COL2A1 gene, in our opinion both family history, clinical data, cell culturing, ultra-structural and immunohistochemistry results suggest that patella alta and trochlea dysplasia is associated with abnormal type II collagen heterofibrils and an ERSD phenotype.

\section{Future Research Directions}

Biopsies from additional patients with similar conditions are needed and further molecular work is required to elucidate the full mechanism for this condition which displays a mild cartilage disorder within the clinical spectrum of chondrodysplasia and to confirm that this phenotype might develop from a specific mutation, which in the first stage could be a novel subfamily within the collagenopathies, with pathology primary confirmed to patella-femoral joint.

This family study has important implications for future research directions of patellofemoral disorders such as patella alta, trochlea dysplasia and chondromalacia patella.

\section{Acknowledgements}

The authors thank Diana Thomsen for her contribution to this manuscript. Study reported here was financially supported by Interface Biotech A/S, Hørsholm, Denmark. None of the authors have conflict of interest in the study.

\section{References}

[1] Snead, M.P. and Yates, J.R. (1999) Clinical and Molecular Genetics of Stickler Syndrome. Journal of Medical Genetics, 36, 353-359.

[2] Spranger, J., Winterpacht, A. and Zabel, B. (1994) The Type II Collagenopathies: A Spectrum of Chondrodysplasias. European Journal of Pediatrics, 153, 56-65.

[3] Skagen, P.S., Horn, T., Kruse, H.A., Stærgaard, B., Rapport, M.M. and Nicolaisen, T. (2010) Osteochondritis Dissecans (OCD), an Endoplasmic Reticulum Storage Disease (ERSD): A Morphological and Molecular Study of OCD Fragments. Scandinavian Journal of Medicine \& Science in Sports, 21, e17-e23.

[4] Kim, P.S. and Arvan, P. (1998) Endocrinopathies in the Family of Endoplasmic Reticulum (ER) Storage Diseases: Disorders of Protein Trafficking and the Role of ER Molecular Chaperones. Endocrine Reviews, 19, 173-202.

[5] Andersen Thestrup, P. (1936) Luxafio Patellus (in Danish, with an English summary) Thesis. Copenhagen.

[6] Ahlbäck, S. and Mattsson, S. (1978) Patella Alta and Gonarthrosis. Acta Radiol Diagn (Stockh), 9, 578-584.

[7] Lancourt JE \& Cristini JA (1975) Patella Alta and Patella Infera. Their Etiological Role in Patellar Dislocation, Chondromalacia, and Apophysitis of the Tibial Tubercle. J Bone Joint Surg Am. Dec;57(8):1112-5.

[8] Chaussé, D.J., Vignes, L. (1982) Critical Study of Patella Alta. II. Patella Alta and Femoropatellar Pathology, 49, 507513.

[9] Jacob, R.P., Gumppenberg, S. and Engelhardt, P. (1981) Does Osgood-Schlatter Disease Influence the Position of the Patella? JBJS British, 63-B, 579-582.

[10] Caton, J., Mironneau, A., Walch, G., Levigne, C. and Michel, C.R. (1990) Idiopathic High Patella in Adolescents. Apropos of 61 Surgical Cases. Revue de Chirurgie Orthopédique et Réparatrice de l'appareil Moteur, 76, 253-260.

[11] Dejour, D. and Le Coultre, B. (2007) Osteotomies in Patello-Femoral Instabilities. Sports Medicine and Arthroscopy Review, 15, 39-46. http://dx.doi.org/10.1097/JSA.0b013e31803035ae

[12] Schulthess, W. (1899) Zur Pathologie und Therapie der Spastischen Gliederstarre. Zur operativen Behandlung der Skoliose, 6, 1-13.

[13] Insall, J. and Salvati, E. (1971) Patella Position in the Normal Knee Joint. Radiology, 101, 101-104.

[14] Nishimura, G., Haga, N., Kitoh, H., Tanaka, Y., Sonoda, T., Kitamura, M., Shirahama, S., Itoh, T., Nakashima, E., Oha- 
shi, H. and Ikegawa, S. (2005) The Phenotypic Spectrum of COL2A1 Mutations. Human Mutation, 26, 36-43. http://dx.doi.org/10.1002/humu.20179

[15] Godfrey, M., Keene, D.R., Blank, E., Hori, H., Sakai, L.Y., Sherwin, L.A. and Hollister, D.W. (1988) Type II Achondrogenesis-Hypochondrogenesis: Morphologic and Immunohistopathologic Studies. The American Journal of Human Genetics, 43, 894-903.

[16] Shabshin, N., Schweitzer, M.E., Morrison, W.B. and Parker, L. (2004) MRI Criteria for Patella Alta and Baja. Skeletal Radiology, 33, 445-450.

[17] Karadimas, J.E., Piscopakis, N. and Syrmalis, L. (1981) Patella Alta and Chondromalacia. International Orthopaedics, 5, 247-249. http://dx.doi.org/10.1007/BF00271078

[18] Yang, L., Carlson, S.G., McBurney, D. and Horton, W.E. (2005) Multiple Signals Induce Endoplasmic Reticulum Stress in Both Primary and Immortalized Chondrocytes Resulting in Loss of Differentiation, Impaired Cell Growth, and Apoptosis. The Journal of Biological Chemistry, 2, 31156-31165.

[19] Rick, A. and Crick, F.H. (1961) The Molecular Structure of Collagen. Journal of Molecular Biology, 3, 483-506. 